\title{
The SN1987A Environment
}

\section{By LIF A N W A NG ${ }^{1}$ AND E. JOSEPH W AMPLER}

\author{
${ }^{1}$ Beijing Astronomical Observatory, Beijing 100080, P.R. China \\ ${ }^{2}$ European Southern Observatory, Karl-Schwarzschild-straße 2, Garching bei München, \\ D-85748 Germany
}

The environment of the SN1987A is quite complex but also very regularly structured. Detailed analyses of direct images taken under good seeing conditions $(0.3-0.8$ arcsec) from the European Southern Observatory (ESO)'s New Technology Telescope (NTT) show that there are two nebular loops within the 3 arcsec environment of the SN. The inner loop is elliptical in shape. The kinematics of this loop as revealed by spectroscopic data with a spectral resolving power $\lambda / \Delta \lambda \approx 30000$ provide further clues for the three dimensional structure of these two loops. The data show that the overall structure of the nebulosity can be understood by an hourglassshaped shell with significant mass enhancement on its equatorial plane. A diffuse nebulosity called Napoleon's Hat is observed at a distance of about 5 arcsec to the north of the SN. It showed little size evolution since the first observation on Aug. 1989, until it disappeared on Jan, 1992. The Napoleon's Hat nebula appears to be a bow-shock coming from an interaction between the supernova progenitor's stellar wind and the interstellar medium, as the supernova progenitor moved through the interstellar medium with a velocity of around $5 \mathrm{~km} \mathrm{~s}^{-1}$. On an even larger scale, there is a huge dark bay of size around 100 arcsec in diameter, we suggested that this bay was also formed by interactions between the supernova progenitor and the interstellar medium.

The narrow emission lines indicate that the electron density and temperature of the emission gas in the loops are steadily decreasing. However, the temperatures inferred from the [OIII] nebular lines remained persistently higher than $2 \times 10^{4} \mathrm{~K}$. They were around $10^{4} \mathrm{~cm}^{-3}$ and $2 \times 10^{4} \mathrm{~K}$ on Aug. 30,1990. In addition, a global difference in the electron density of the two major axis of the inner loop is observed in the images and spectra taken five years after the supernova explosion. We have also studied the two neighboring stars surrounding the supernova. We find that star 2 is a $\mathrm{B}$ star with $H_{\alpha}$ and $H_{\beta}$ emission lines.

The supernova circumstellar nebulosity is remarkably similar to certain types of planetary nebula, e.g. NGC2392. Asymmetric wind interaction models for the formation of planetary nebula are applied to produce a model in the form of an hourglass shaped shell. This model invokes both a slow wind while the star was in its red supergiant phase and a fast wind during the later blue supergiant stage before explosion. Although such wind interaction models fit the observed nebular structures satisfactorily, they are so idealized that they tell us little about the origin of the seed asymmetry or the amount of seed asymmetry needed to produce the observed real nebula.

\section{Introduction}

The first evidence of circumstellar gas near SN1987A was the detection, starting on about day 80 , of narrow nebular lines in the $I U E$ spectra (Wamsteker et al, 1987; Fransson et al. 1989) . Narrow optical lines were first seen on about day 300 (Wampler \& Richichi 1989). The UV and optical data gave early information of the size, density, electron temperature of the brightest part of this nebula (Fransson et al 1989; Wampler \& Richichi 1989). Later observations began to reveal in more detail the structures of the circumstellar material (Wampler, Richichi \& Baade 1989; D'Odorico \& Baade 1989; Crotts, Kunkel, \& McCarthy 1989, Sparks, Paresce, \& Maccheto 1989).

Using observations collected at the European Southern Observatory, we discuss the 
structures seen in the SN1987A circum-stellar nebulosity. In section 2, we discuss the structures on different scales, namely, the Nebular loops, the Napoleon's Hat Nebula, and the Dark Bay. At the end of this section, we show that one of the stars near the SN is a B star with emission lines. In Section 3 we briefly review the existing models for the nebular loops and other structures on a larger scale. Finally, we summarize in section 4 .

\section{Observations of the SN1987A Environment}

We base our discussions mainly on observations collected at the European Southern Observatory's New Technology Telescope, and compare these observations with those obtained by other authors. We discuss the geometrical structure of the nebulosity as well as its physical parameters such as electron density and temperature.

\subsection{The Nebular Loops}

The first ESO NTT images of the supernova, taken on Dec. 1989, show that the supernova is surrounded by a regularly structured nebulosity which is remarkably similar to certain planetary nebulae (Wampler et al, 1990, hereafter W90). As shown in Fig. 1, it basically consists of a central oval with two much fainter loops connected to the central oval. This nebulosity is conspicuous in both narrow band filters which emphasize nebular line emissions and in broad-band filters that emphasize continuum emissions. By comparing the size of this nebulosity with previous observations, W90 found little change of the size of these nebula. This behavior is quite different from the outer two rapidly expanding large light echoes (Crotts, et al; Gouiffes et al, 1989). These observations excluded earlier suggestions made by Crotts et al (1990) that the observed structures are due to light echoes of a sheet of background material, and W90 concluded that the observed structure represents the real three-dimensional structure of the nebulosity.

The details of the inner oval described in W90 are shown in an excellent HST image (Jacobson et al, 1991; Panagia et al, 1991). It reveals an elliptical ring whose major and minor axes are $1^{\prime \prime} .66 \pm 0^{\prime \prime} .03$ and $1^{\prime \prime} .21 \pm 0^{\prime \prime} .03$, respectively. Jakobsen et al (1991) argue that, because there is little emission detected at the center of the ring, the elliptical ring must be the projection of a real circular loop inclined by about $43^{\circ}$ to the line of sight. However, the HST image has a very low dynamic range; it fails to show the outer loop which is only about ten-twenty times fainter, so the pure ring geometry for the nebular ring is only an approximation.

If, as W90 argued, the observed structure is due to the projection of a definite physical structure onto the plane of the sky, we can infer a shell geometry that is similar to an hourglass. This model is shown in Fig. 2. In this picture, the inner ellipse is due the waist of the hourglass shell and the outer loop is due to the two lobes of the shell which are limb-brightened when projected to the plane of sky. The distance from the symmetry center of the hourglass shaped shell to its waist is about $1 / 3$ of the distance to the poles (Wang \& Wampler, 1992). The density distribution across the shell is not homogeneous; there must be a strong density enhancement on the equatorial plane of the shell to account for the bright inner ring. Slit spectra show that the nebula is expanding at velocities about $10.3 \mathrm{~km} \mathrm{~s}^{-1}$ (Crotts \& Heathcote, 1991); these kinematics are consistent with the geometry given in Fig. 2.

Both the electron temperature and the electron density of the emission-line region have been found to be decreasing since the detection of the nebula. The highest electron density is estimated from IUE satellite observations (Fransson et al, 1989), and from the optical observations of Wampler \& Richichi (1989), and Wampler, Richichi \& Baade (1989). The decrease is due apparently to radiative cooling and recombination after 

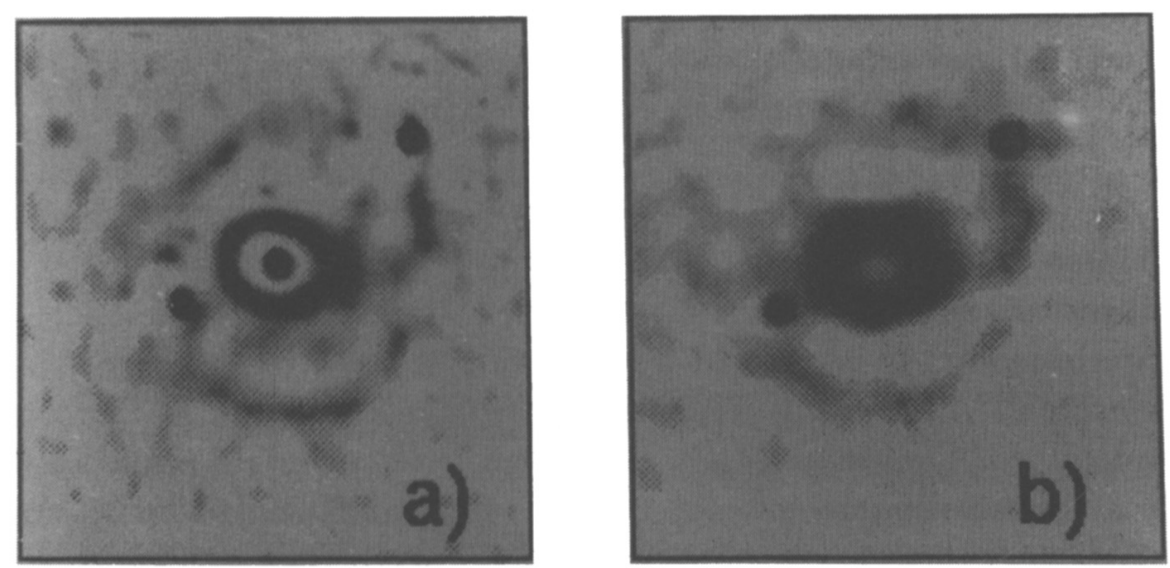

FIgURE 1. [OIII] Images of the nebular loops: (a) Day 1037, and (b) day 1790 after the SN explosion.

the nebulosity was ionized by the initial UV flash from the supernova outburst. Wang (1991) noticed the temperature of the nebulosity is not decreasing as rapidly as predicted by model calculations by Lundqvist \& Fransson (1991), and suggested that a mixed structured of different electron density can explain this behavior: at early times we observe mostly the dense part of the nebula which cools faster than the less dense part; but eventually, the dense part has completely recombined and emission from the low density part dominates the emissions. Later models with several density component yields better agreement with observations (Luo, 1991).

Although the inner ellipse is very regular, the distribution of electron densities along the ellipse is probably not homogeneous. Images taken after Jan. 1991 show a clear difference in brightness of the East and West side of the ring. Spectroscopic data taken with slit across the East and West side of the ring show that the density sensitive [SII] $671.6,673.1 \mathrm{~nm}$ line ratio is not the same on the two sides of the ring, with the electron density of the west side being lower than the east side. A natural explanation is that the west side, having lower electron density than the east side, therefore cools more slowly, and is now brighter than the east side.

\subsection{Napoleon's Hat}

The Napoleon's Hat nebula refers to a diffuse nebulosity to the north of the SN. It was conspicuous in images taken in continuum filters but was only weakly seen in images taken using narrow line filters. Wang \& Wampler (1992) compared images taken from Aug., 1989 to Jan. 1992, and found little change in size of the northern extension of this nebulosity, although they detected expansion to the east and west side. It thus seems that the majority of the nebulosity responsible for the Napoleon's Hat nebula was already inside the light travel paraboloid defined by the equal light travel time from the $\mathrm{SN}$ to the observer as shown in Fig. 2. It follows that the nebulosity can not be a clump of dusty gas located far from the supernova and along the line of sight to the supernova; its location is strictly constrained to be near the SN (Wang \& Wampler, 1992).

Two possibilities were proposed for Napoleon's Hat : 1) a bow-shock model; 2) a tilted disk model (Wang \& Wampler, 1992). The bow-shock model gives better fit to the data, 


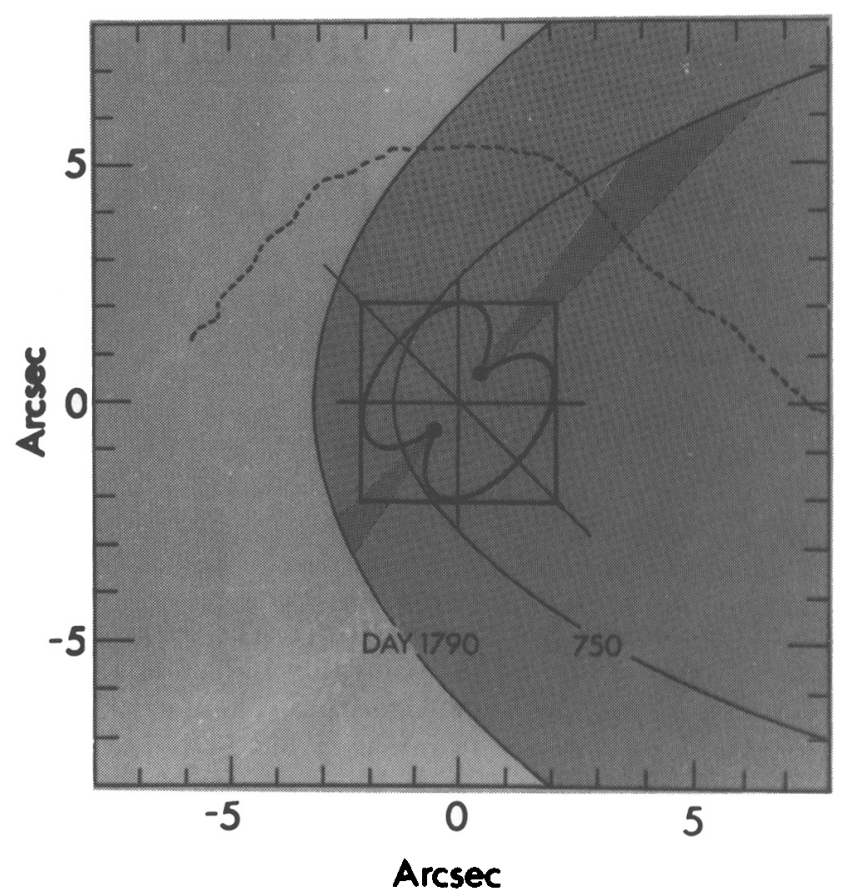

Figure 2. Geometry for the Nebular loops and Napoleon's Hat. The light travel paraboloids of the SN light are shown for different dates after the SN explosion. The central box shows the allowed location for the nebulosity responsible for the two nebular loops. Also shown are the observed shape of Napoleon's Hat (Dotted lines) and the hypothesized disk. North is up, right is to the direction of the observer.

while the disk model gave only a rough fit to the size and shape of the light echo when it was first seen. Moreover, it does not at all reproduce the time evolution of Napoleon's Hat, and, in particular, that part that is the top of the Hat. However, a combined structure of a disk and a bow shock may be a possible model for the Napoleon's Hat, especially when considering the details of the brightness distribution across the observed diffuse nebulosity of the Napoleon's Hat. Although we lack firm evidence for a disk, it could provide a natural link between the Napoleon's Hat and hourglass shaped shell.

\subsection{Structures beyond 5 arcsec}

Several larger echoes have been detected near SN1987A. The most spectacular of these are the two echoes discovered by Crotts (1987) and described by Rosa et al (1987). These echoes are rapidly expanding, and it is clear that they are due to reflections of the supernova light by two dusty sheets lying between us and the supernova at distances of $105 p c$ and $316 p c$ from the SN. They show that the SN lies behind two curtains of dusty material, but tell us very little about the supernova progenitor.

A light echo ring of size 10 arcsec was detected on Jan. 1989 (Bond et al, 1990; Sparks et al, 1990; and Crotts \& Kunkel, 1991) The echo was interpreted by Chevalier \& Emmering (1989) as due to the shock wave arose from the interactions between the supernova progenitor's RSG wind and surrounding interstellar medium. However, this light echo was observed only briefly; it was neither detected in our NTT images taken in 1990 Aug. nor afterwards; this would suggest that the reflecting material is probably 


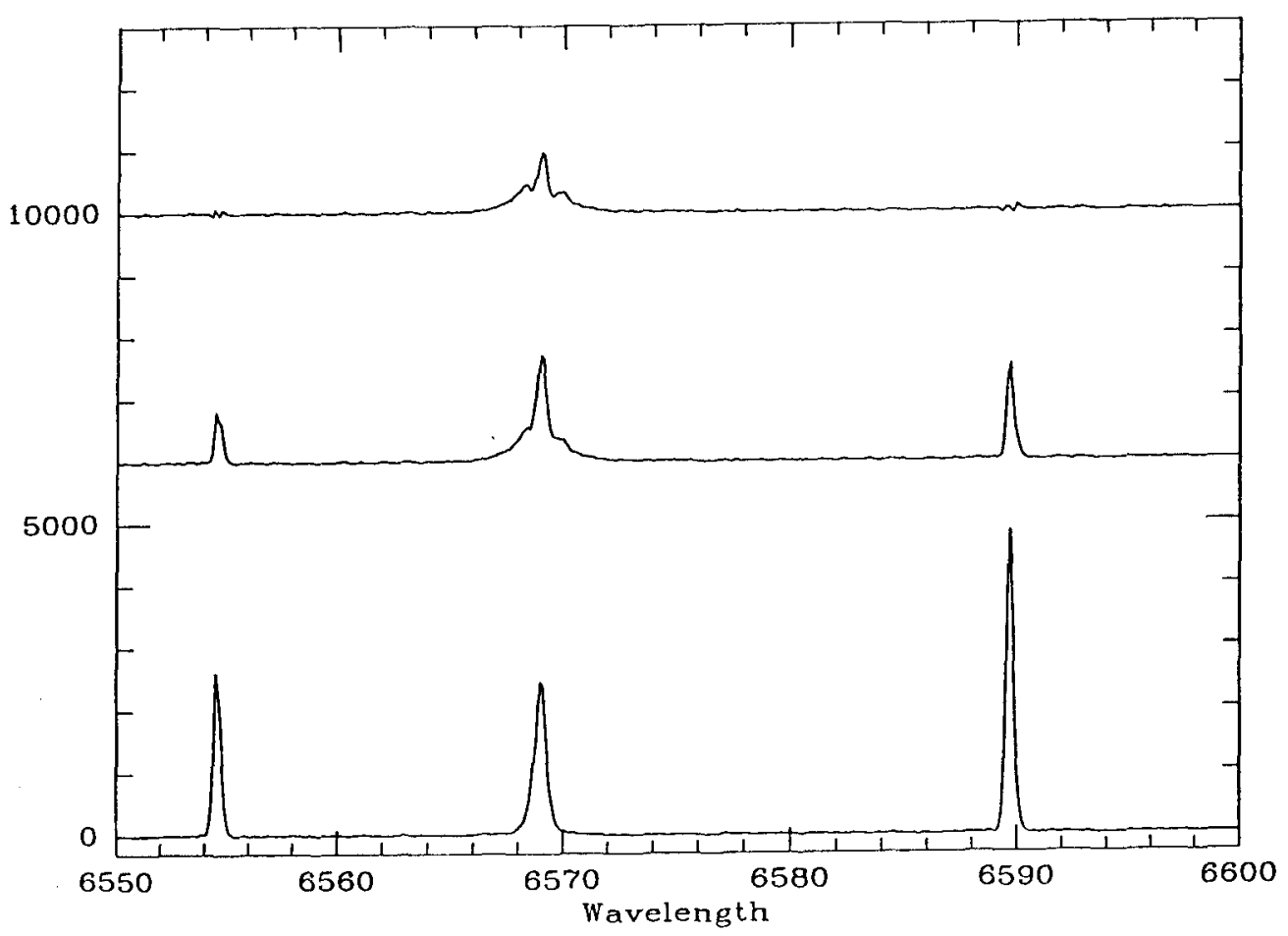

Figure 3. The spectrum of star 3 near SN1987A.

not a closed shell of gas centering at the supernova, but is simply a dusty clump in front of the supernova.

\subsection{Star 3}

Since massive stars are usually found in associations, it is interesting to study the stars in the supernova neighborhood. There are two blue stars close to the supernova. We show in Fig. 3 the spectra for star 3, which is located at position angle $110^{\circ}$ and 1.5 arcsec away from the supernova. The spectra shown were obtained at the NTT with the ESO Multiple Mode Instrument (EMMI) on Jan. 15, 1993. For the exposure, star 2, the SN and star 3 were simultaneously within the slit. We see a clear broad $H_{\alpha}$ component close at the position of star 3. This broad component is not seen in the metal lines (Wang et al, 1992). We have compared our NTT direct images taken at seeing conditions of around 0.3 arcsec, and found that the star 3 is brighter than star 2 in the narrow band $H_{\alpha}$ filter, but is much fainter in other bands.

We have tried to construct spectra of star 2 ; we subtracted the contamination by the supernova light by removing the neighboring [NII] lines completely. In the subtracting process, we scaled the [NII] $658.4 \mathrm{~nm}$ line so that in the subtracted spectra this line completely disappears. In doing so we found that the [NII] $654.8 \mathrm{~nm}$ line also disappeared automatically. The resulting spectra show clearly a broad $H_{\alpha}$ feature. Combining the above observations with the CTIO photometric data which show star 3 is variable (Elias et al, 1993), we conclude that the emission feature is intrinsic to star 3. The HST images should be able to provide further constraints on the nature of this broad emission feature; this has not been done so far, however. 


\section{Dynamical Models}

\subsection{The Slow-Fast Wind Interaction Model for the Nebular Loops}

Luo \& McCray (1990), Wang (1991), Blondin \& Lundqvist (1992) proposed that the nebular loops can be modeled in terms of the asymmetric slow and fast wind interaction models used to explain the morphological development of planetary nebulae (Kahn \& West, 1985). The model assumes that SK -69 202 was once a RSG before it evolved to a BSG and exploded. In the RSG stage, the star loses mass at a rate of typically $10^{-5} M_{\odot} /$ year and velocity around $10 \mathrm{~km} \mathrm{~s}^{-1}$; while in the BSG stage, the mass loss rate decreased by about 10 times to $10^{-6} M_{\odot} /$ year, but the wind is much more energetic; the wind velocity is typically about $10^{3} \mathrm{~km} \mathrm{~s}^{-1}$.

An important parameter in this model is the amount of the initial asymmetry in the slow RSG wind. Luo \& McCray (1990) used a ratio for the pole to equator mass loss rate of about 1:5, while Wang \& Mazzali (1992) used a ratio of only 1:1.2. Wang \& Mazzali found that, even with the very small asymmetries they used, a high density cusp forms at the equator of the bubble of the shocked slow wind, and this cusp can account for the observed structure.

The thin-shock approximation allows for a similarity solution (Kahn \& West, 1985); this similarity solution, however, breaks down in the region where the cusp develops. In the thin shock approximation, the surface density of the shocked slow wind shell is determined by two effects: 1) the dilution due to radial expansion and 2) the forced non-radial motion due to the pressure in the shocked hot bubble. A cusp will develop if the compressed motion overtakes the dilution by expansion. An analytical criteria is given by Wang (1993) for the formation of a high density cusp.

The details of cusp formation must be studied by hydrodynamic simulation. Such calculations were made by Blondin \& Lundqvist (1993). Their best fit model has a high concentration of mass loss rate on the equatorial plane of the progenitor star. Their hydrodynamic model fits not only the size of both the inner and outer loops, but also predicts the clumpy structure observed recently by the NTT image for both loops (W90, Wang \& Wampler, 1992) and by the HST images for the inner ellipse (Jacobsen et al, 1991; Panagia et al, 1991).

Though it seems that these models successfully reproduce structures observed around the supernova, the uniqueness of the models are not guaranteed. An important problem is that the observed expansion velocity of the inner ellipse is only about $10 \mathrm{~km} \mathrm{~s}^{-1}$, a value that is less than typically observed RSG wind velocities. Although the formation of high density cusps can be achieved in thin-shock approximation models they can not be used to estimate the amount of the initial asymmetry in the slow wind. The models fail when the cusps develops; the cusp is a real mathematical critical point. This explains why the Luo \& McCray (1991) and the Wang \& Mazzali (1992) models used different values for the seed asymmetry in the slow wind. Neither author attempted to estimate the exact amount of asymmetries required to produce the supernova nebular shell. More realistic considerations of the wind interactions remain to be explored.

One recent improvement (Wang, 1993) is to assume that the slow wind mass loss rate is not steady; variable mass loss rates are believed to be responsible for the zoo of planetary nebulae morphologies, after the early normal RSG wind stage and before the BSG wind starts there may be a super wind stage. We have run a hydrodynamic code SADIE (Arnold, 1985) kindly provided by E. Müller; the model results are shown in Fig. 4. The model parameters are taken to be similar to those of Wang \& Mazzali (1992), but the mass loss rate of the slow wind is assumed to have a sharp jump by a factor of ten. 


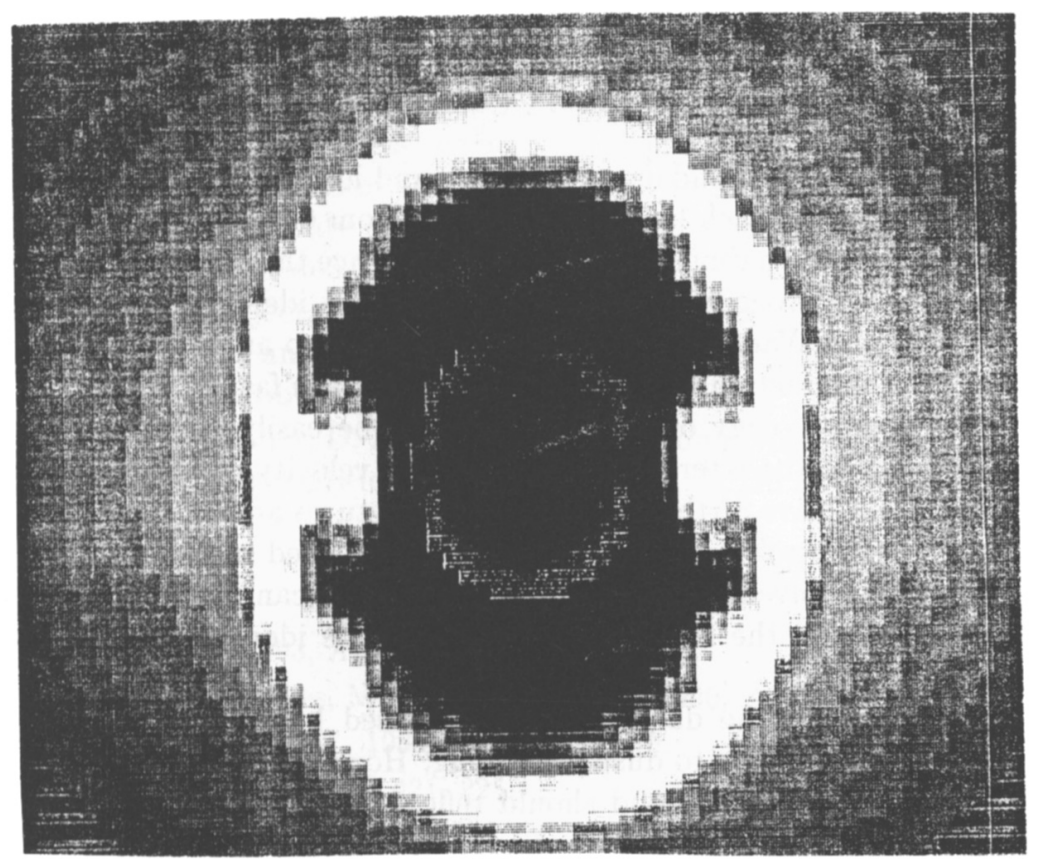

Figure 4. Models with non-steady slow winds

It is readily seen that the final structure deviates more strongly from spherical symmetry in the non-steady slow wind scenario than in the steady slow wind scenario, and thus a reduction in the amount of initial asymmetry required. In the steady slow-wind scenario, the enhancement of materials on the equatorial plane is determined by the nonradial motions onto the equator. On the other hand, in the super wind scenario, the hot bubble and therefore the shocked slow wind shell expands faster in the polar direction than in the equatorial plane. It breaks through the dense superwind first at the two poles, there the ram pressure of the slow wind decreases sharply and the shell is accelerated by the pressure of the hot bubble. On the equatorial plane, this process happens later. By that time, the pressure of the hot bubble is greatly reduced due to expansion in the polar direction. We note that the resolution in our model calculation is rather low; higher resolution calculations should reveal more structural details. The model can possibly resolve the polar caps observed in many planetary nebulae.

One other possibility was proposed recently by Chevalier \& Luo (1994), who considered the shaping due to magnetic field; they also found the formation of an equatorial cusp.

\subsection{The Effect of the RSG and the Main Sequence Wind}

It is believed that the progenitor star started as a BSG star on the main sequence and that it later evolved to a RSG star and then to a BSG before exploding (Nomoto et al, 1987; Woosley, 1988). Since massive stars are believed to have stellar winds during their entire life time, these pre-supernova stages can also be effective in forming nebular structures. Wang \& Wampler (1992) find that the Napoleon's Hat is the next neighboring structure outside the two nebular loops. The location of the material responsible for the Napoleon's Hat is strictly constrained by the time evolution of the Napoleon's Hat. The Napoleon's Hat is so close to the SN that one can safely say that this nebulosity must be dynamically related to the supernova progenitor. The next structure outside the 
Napoleon's Hat nebula is the echo ring at a radius of 10 arcsec. But, if this echo is due to the shell identified with the shock coming from the interaction between the slow wind and the bubble of the reverse shock of the first BSG wind, the echo should be still observable. It is not.

It is difficult to find the boundary of the fast wind lost by the progenitor while it was in its first BSG stage. On such large scales, winds from other associated stars and even supernova explosions that happened earlier may influence the environment into where the wind of the supernova progenitor expanded. A possible identification of this boundary was given by W90 and Wang, Kahn \& Dyson (1993), who argue that the dark bay can be the result of the interaction between the main sequence fast wind and the interstellar medium, and the morphology of the dark bay can be easily understood if the star is moving with respect to the interstellar medium at a velocity of about $5 \mathrm{~km} \mathrm{~s}^{-1}$. Wang, Dyson \& Kahn (1993) have further modeled the structures arising from such interaction, and find that the star itself can move through the shocked interstellar medium by the time the progenitor evolved to a RSG; the RSG wind can interact directly with the interstellar medium, and the resulting structure can be identified with the Napoleon's Hat nebula.

Another possibility for the dark bay was proposed by Felten \& Dwek (1991) who argue that the dark bay is due to dust shadowing. However, we think this interpretation is unlikely. Even a very dusty cloud should reflect enough supernova light to produce observable light echoes. The two observed echo rings have already expanded to a larger angular size than the dark bay (Wang \& Rosa, 1992) and cannot be reflections from the hypothesized dust cloud. No nebulosity has been found that can be attributed to reflections from the shadowing dust cloud.

\section{Summary}

We have shown that the environment of the supernova SN1987A is strongly influenced by the wind from the progenitor star. Therefore, the structure of the SN1987A environment provides valuable information about the supernova progenitor. The two nebular loops are identified with the shock arising from the interactions between the progenitor's blue and red supergiant wind. To understand the hourglass morphology, one must assume certain asymmetries in the progenitor's stellar winds. The origin and degree of this asymmetry is not clear: it may be due to a binary star if the degree of asymmetry is large; but a single star model may also be possible if only a small degree of asymmetry is required.

The Napoleon's Hat nebula to the north of the nebular loops can be best fit by a bowshock. A disk model does not give the right observed morphology at late times. But there are some weak indications of the existence of a disk from the brightness distributions on the surface of Napoleon's Hat in the Dec. 1989 observations. The bow-shock/disc is the next structure outside the nebular loops, and it is affected by the winds from the SN progenitor. A natural explanation is that the progenitor moves through the interstellar medium, and its wind interacts with the surrounding interstellar medium and produces the bow-shock.

The 10 arcsec scale light echo does not seem to be due to a shell enclosing the supernova, but most likely is a dusty clump that happens to be located along the line of sight to the $\mathrm{SN}$. The structure of the Dark bay is much more complex, but may also be influenced by the wind from the supernova progenitor.

Hydrodynamic models for the nebular loops have to reproduce the following three observed properties: (1) the ratio of the radius of the inner and outer loop is about 
$1: 3 ;(2)$ the ratio of the emission measure of the inner and outer loop should be larger than 10; and (3) the expansion velocity of the inner ring should be about $10 \mathrm{~km} \mathrm{~s}^{-1}$. Models with steady mass loss rate in the BSG an RSG stage have been studied in great detail, but more complicated models seem to be required. A major modification and complication would be to abandon the steady wind assumption. Our low resolution hydrodynamic simulation already shows that this is a promising approach, but higher resolution simulations are needed.

Finally, the supernova ejecta are expanding at velocities up to $30000 \mathrm{~km} \mathrm{~s}^{-1}$. The collision between the ejecta and the circum-stellar material will excite the gas again, and further information can be obtained when this occurs.

We are grateful to R. Chevalier, D. Baade, L. Lucy, P. Lundqvist, D. Luo and K. Nomoto for discussions. L. W. also acknowledges Q. B. Li and J. Y. Hu for supporting this work.

\section{REFERENCES}

Blondin, J. \& Lundqvist, P. 1993, ApJ, 405, 337

Bond, H. E., Gilmozzi, R., Meakes, M. G., \& Panagia, N. 1990, ApJL, 347, L65.

Chevalier R. A. \& Emmering, R. T., 1989, ApJL, 342, L75.

Chevalier R. A. \& Luo, D., 1994, ApJ, 421, 225.

Crotts, A. P. S. 1988. IAU Circ. 4561

Crotts, A. P. S., \& Heathcote, S. R. 1991, Nature, 350, 683.

Crotts, A. P. S., \& Kunkel, W. E. 1991, ApJL 366, L73.

Crotts, A. P. S., Kunkel, W. E., \& McCarthy, P. J. 1989, ApJL, 347, L61.

D'Odorico, S., \& Baade., D. 1989, ESO Messenger, 56, 35.

Elias, J. H., Phillips, M. M., Suntzeff, N. B., Walker, A. R., Gregory, B., \& Depoy, D. L. 1993, in Massive Stars: Their lives in the Interstellar Medium, Ed. Cassinelli, J. P. \& Churchwell, E. B., P408.

Felten, J. E. \& Dwek, E., in SN1987A and Other Supernovae, ed. Danziger, I. J. \& Kjä r, K. P569.

Fransson, C., Cassatella, A., Gilmozzi, R., Kirshner, R. P., Panagia, N., Sonneborn, G., Sauvageot, J. L., \& Wamsteker, W., 1989, $A p J, 336,429$.

Gouiffes, C., Rosa, M., Melnick, J., Danziger, I. J., Renny, M., Santini, C.,Sauvageot, J. L., Jakobsen, P., \& Ruiz, M. T., 1988 A $\mathcal{B} A$ 198, L9

Jakobsen, P., et al $1991 A p J L$, L63.

Kahn, F. D., \& West, K. A. $1985 M N R A S, 212,837$.

Luo, D., 1992, Ph. D. thesis, University of Colorado.

Luo, D., \& McCray, R. 1991, ApJ, 379, 659.

Lundqvist, P., \& Fransson, C. 1991, ApJ, 380, 575.

Meikle, W. P. S., Cumming, R. J., Spyromilio, J., Allen, D. A., \& Mobasher, B. 1990, in Proceedings of the ESO/EIPC Workshop on SN 1987 A and other Supernovae ed. I. J. Danziger, p595.

Nomoto, K., Shigeyama, T., Hashimoto, M., in Proceedings of the ESO/EIPC Workshop on SN 1987A and other Supernovae ed. I. J. Danziger, p325.

Panagia, N., Gilmozzi, R., Macchetto, F., Adorf, H.-M., Kirshner, R.P. 1991, ApJL, 380, L23.

Sonneborn, G., \& Wamsteker, W. 1989, ApJ, 336, 429.

Sparks, W. B., Paresce, F., \& Macchetto, D. 1989, ApJL, 347, L65.

Wampler, E. J., \& Richichi, A. 1989, $A \& A$, 217, L21.

Wampler, E. J., Richichi, A., \& Baade, D. 1989, in IAU Colloquium 120, Structure and Dynamics of the Interstellar Medium, ed. G. Tenorio-Tagle, M. Moles, \& J. Melnick (Berlin-Springer), 
P180.

Wampler, E. J, Wang, L., Baade, D., Banse, K., D'Odorico, S., Gouiffes, C., \& Tarenghi, M. 1990, ApJL, 362, L13 (W90).

Wamsteker, W., Gilmozzi, R., Cassatella, A., \& Panagia, N. 1987, IAU Circ, No. 4410.

Wang, L. 1991, $A \& A, 246$, L69.

Wang, L., D'Odorico, S., \& Wampler, E. J. 1992, IAU Circ. 5449.

Wang, L., Dyson, J. E., \& Kahn, F. D. 1993, MNRAS, 261, 391.

Wang, L., \& Mazzali, P. A. 1992, Nature, 355, 58.

Wang, L., \& Rosa, M. 1992, ESO Messenger, 67, 37.

Wang, L. \& Wampler, E. J. 1992, $A \& A, 262$, L9.

Woosley, S., 1988, ApJ, 330, 218. 